\title{
Avaliação de reservatório carbonático da Bacia de Campos integrando dados de laboratório com perfis de poço
}

George Nocchi ${ }^{*}$, Abel Carrasquilla*, Nelson Franco Filho** \& Frederico Schuab**

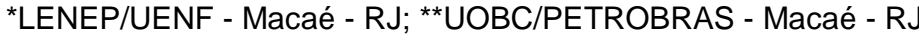

Copyright 2012, SBGf - Sociedade Brasileira de Geofísica

Este texto foi preparado para a apresentação no V Simpósio Brasileiro de Geofísica, Salvador, 27 a 29 de novembro de 2012. Seu conteúdo foi revisado pelo Comite Técnico do V SimBGf, mas não necessariamente representa a opinião da SBGf ou de seus associados. É proibida a reprodução total ou parcial deste material para propósitos comerciais sem prévia autorização da SBGt.

\section{Resumo}

In this study, a carbonate reservoir in Campos Basin was evaluated through open hole logs and laboratory petrophysical data of three wells. Initially, conventional logs were used to identify the boundaries of the reservoir and the oil-water contact. After this, laboratory data was used to divide the reservoir into lithofacies, petrophysical areas as well as indicators of reservoir quality. In the same sense, the flow zones method was used and its results were compared with the production log data, thereby evaluating its effectiveness. On the other hand, Winland's graph was utilized to assess the best petrophysical quality areas and the regions of the reservoir that had similar flow indicators were classified as flow units of the reservoir. Each flow unit was fitted by a linear equation, which allowed extrapolation to other wells and the estimation of the permeability. Moreover, the Nuclear Magnetic Resonance (NMR) log was essential to derive petrophysical information in the wells, as macro, meso and micro porosity. For each flow zone were generated $\mathrm{T}_{\text {2cutoff, }} \mathrm{BFT}, \mathrm{FFI}, \mathrm{S}_{\text {wir }}$, permeability and the pseudo capillary pressure curves. The logs were calibrated with different laboratory analysis including mercury injection capillary pressure (MICP) and, thus, NMR allowed an appropriated fit between empirical equations and the data of the analyzed formation. The $T_{2}$ distribution obtained by NMR laboratory also was enabled to evaluate the wettability of the formation. Thus, in the description the petrophysical conditions of the reservoir in each well, it was possible to discover the potential flow areas, providing the information that feeds the geological models necessaries to simulate the behavior of reservoir.

\section{Introdução}

As rochas reservatório, além de possuírem um sistema poroso suficiente para conter grandes quantidades de hidrocarbonetos, devem ainda possuir poros bem conectados entre si, de modo que os fluidos possam circular pela rocha e serem produzidos. As propriedades petrofísicas das rochas ao longo do poço podem ser analisadas de modo direto, através de amostras de calha, plugs de testemunho, análise de fluido de perfuração e teste de pressão, ou por métodos indiretos, como a perfilagem.

A maioria dos reservatórios de hidrocarbonetos no mundo se encontra em rochas carbonáticas, Estes, ao contrário dos reservatórios siliciclásticos, sofrem um complicado processo diagenético, que inclui cimentação, dissolução, dolomitização, recristalização e ação biogênica. Devido à essa complexidade litológica, as rochas carbonáticas, são de difícil caracterização, exigindo assim estudo e experiência (Lucia, 1999).

A Bacia de Campos (Figura 1) possui mais de 1.600 poços perfurados ao longo de mais de três décadas de exploração petrolífera, sendo a bacia brasileira mais prolífica e alojando a maior parte das reservas petrolíferas atuais do país. Recentemente, foi descoberta a maior reserva de hidrocarbonetos do Brasil. Trata-se de um reservatório carbonático, na camada do pré-sal, que se estende por diferentes bacias, incluindo a Bacia de Campos. Desde então um novo desafio surgiu no cenário da exploração petrolífera brasileira, e consequentemente, na área acadêmica, aumentando a necessidade de melhor compreender os reservatórios carbonáticos (Machado et al., 2011).

Neste contexto, este trabalho visa investigar o sistema poroso e gerar resultados que contribuam para exploração e produção de petróleo num reservatório carbonático do pós-sal.

\section{Metodologia}

Os dados utilizados neste trabalho foram extraídos de três poços (P1, P2 e P3) que atravessam um reservatório carbonático da seção pós-sal da Bacia de Campos (Figuras 2). Apesar do reservatório estar localizado numa mesma plataforma carbonática (Figura 4), a variação de fácies sedimentares é grande ao longo e lateralmente dos poços.

Os perfis geofísicos de poço (Tiab \& Donaldson, 1996) utilizados na interpretação foram: caliper - CALI, (in), Raios gama - GR, (gAPI), resistividade (Rt) rasa - LLS, (ohm.m), resistividade profunda - LLD, (ohm.m), sônico DTCO (us/ft), densidade - RHOB ( $\mathrm{g} / \mathrm{cm} 3)$, porosidade neutrônica - NPOR, (\%), NMR: $\mathrm{T}_{2}$ DIST, Schlumberger CMR-Plus 30 bins (ms), NMR: T2LM $_{2}$ média logarítmica dos valores de $\mathrm{T}_{2}$ (ms) e PLT - Production Logging Tool (m3/D).

Medidas diretas de petrofísica básica foram realizadas em 5 amostras laterais do poço P1, em 178 amostras de plugs retirados de testemunhos do poço P2 e em 20 amostras de laterais do poço P3. Medidas de petrofísica especial foram realizadas somente para os poços P2 e P3. O poço P2 é o que apresenta maior número de amostras de testemunho e por esse motivo foi usado como poço de referência, no qual foram desenvolvidos inicialmente os modelos petrofísicos de análise: expansão de gás hélio (petro:perm-h, permeabilidade horizontal (mD) e petro:poros), porosidade total (\%)), intrusão de mercúrio (PcHg) (PcHg:perme, permeabilidade $(\mathrm{mD}), \mathrm{PcHg}$ :poros, porosidade total (\%), $\mathrm{PcHg}$ :Micro, porosidade (\%), PcHg:Meso, porosidade (\%), PcHg:Macro, porosidade (\%)), eletrocapilarômetro 
(equipamento de análise de pressão capilar membrana e propriedades elétricas) (PcMembrana:Swi, saturação de fluido irredutível (\%)), centrífuga (PcCent:Swi, saturação de fluido irredutível (dec)), NMR ( $T_{2 L a b}$ : $S_{w i}$, saturação de fluido irredutível (\%), $\mathrm{T}_{2 \mathrm{Lab}}$ : Cortes $\mathrm{T}_{2}$, valores de $\mathrm{T}_{2 \text { cutoff }}$ (ms), T2Lab: phi_nmr, porosidade total (\%), $\mathrm{T}_{2 \mathrm{Lab}}$ : K_TIMUR, permeabilidade (ms), Timur-Coates $\left(\bar{C}^{\prime}=3.37 \mathrm{E}-05, a^{\prime}=-7.436, b^{\prime}=4,028\right)$.

A primeira etapa do estudo consistiu em analisar os perfis convencionais corridos nos poços, com o intuito de identificar as possíveis zonas de reservatório, assim como as profundidades do topo e da base, além do contato óleo - água. Ao identificar as zonas de reservatório, verifica-se que as curvas dos perfis variam muito. Este fenômeno ocorre devido a complexidade geológica das rochas carbonáticas, e as variações faciológicas, o que dificulta a caracterização petrofisica.

Visando minimizar as dificuldades de análise referentes a heterogeneidade da formação, a região de reservatório foi dividida em zonas que apresentassem propriedades petrofísicas similares. As metodologias usadas para esta divisão foram as de litofácies, zonas petrofísicas (ZP), a qual se baseia nas análises de laboratório e no comportamento das curvas dos perfis. As demais metodologias usadas são baseadas nos conceitos de Amaefule et al. (1993) e Gunter et al. (1997). Após o reservatório ser zonificado de acordo com parâmetros petrofísicos, estes foram confrontados com os dados do perfil de produção - PLT, de modo a identificar a metodologia que melhor representa as zonas de fluxo. Com o reservatório zonificado, tornou-se mais confiável utilizar os dados de perfis e combiná-los com dados de testemunho, de modo a obter as propriedades petrofísicas ao longo do poço. Os perfis convencionais foram então utilizados em conjunto com o perfil NMR, o qual foi a principal ferramenta de análise. Os cortes de $\mathrm{T}_{2}$ foram obtidos por zona, o que permitiu gerar curvas de porosidade efetiva, permeabilidade e saturação de fluido irredutível nas diferentes zonas do reservatório e, por fim, identificar as principais zonas produtoras.

\section{Resultados}

Os perfis convencionais foram essenciais na identificação da zona de reservatório, os seus limites e o contato óleo/água. A Figura 3 mostra como os perfis variam ao longo do poço P2. Desta forma, o perfil litológico GR registrou valores altos até a profundidade de $1.100 \mathrm{~m}$, os quais são característicos em argilas e folhelhos, com uma amplitude da ordem de 75 a 150 unidades API. De um modo geral, espera-se que os carbonatos, por resultarem da matéria esqueletal de vida marinha, possuam os valores mais baixos de radioatividade. A região entre a profundidade de 1.100 e 1.500 metros apresenta baixos valores GR ao redor de $30 \mathrm{API}$.

O perfil Rt dessa região é alto, evidenciando a presença de hidrocarboneto (Figura 3), além disso, a combinação dos perfis de RHOB e NPHI mostra um crossover tipico para regiões reservatório de óleo leve ou gás. Assim, a região de reservatório foi identificada entre as profundidades de 1.100 e $1.200 \mathrm{~m}$ e o contato óleo água em profundidades de 1.178,6 m. A constatação da presença de óleo não é suficiente para transformar a formação atrativa do ponto de vista comercial, o sistema poroso deve ser suficiente para conter grandes quantidades de hidrocarbonetos e, ainda, possuir poros bem conectados entre si, de modo que os fluidos possam circular pela rocha e serem produzidos.

Através da amostragem aleatória de plugs retirados de testemunhos do reservatório foram realizadas as descrições sedimentológicas, assim como as análises de petrofísicas de laboratório para avaliar a porosidade e permeabilidade dos plugs. Constatou-se que a região de reservatório é uma formação carbonática possuindo grande variação litológica (Franco Filho et al., 2011). Devido à heterogeneidade da formação, as curvas dos perfis variam muito impedindo utilizar uma mesma abordagem petrofísica para todo o reservatório ao longo do poço.

A descrição sedimentar realizada através dos plugs de testemunho permitiu identificar oito litofácies, no entanto, para facilitar, estes foram agrupados de acordo com a textura das laminas examinadas pela microscopia e as fotografias de testemunhos, como segue:

- Grainstone 1 e 2 - Grainstone oolítico / microoncolítico, médio a grosso, com raros bioclastos e pelóides. Apresenta porosidades do tipo intergranular, intercristalina, vugular e intragranular (dissolução de grãos). Representa as fácies de alta energia com menor permeabilidade.

- Grainstone 3, 4, 5 e 6 - Grainstone oolíticomicrooncolítico/Rudstone oncolítico, grosso, granulosos, com pelóides finos. A porosidade é do tipo intergranular e vugular, de maiores permeabilidades.

- Rudstone/Grainstone 7 - Rudstone a rodolitos de algas vermelhas, de diâmetro entre 2 e $3 \mathrm{~cm}$, imersos em matriz de grainstone oncolítico granuloso, microoncolítico muito grosso a grosso, e fragmentos de algas vermelhas. A porosidade também é do tipo intergranular e vugular.

- Packstone 8 - Packstone oolítico/oncolítico, peloidal e bioclástico. No espaço poroso são predominantes as porosidades do tipo intergranular e vugular.

A Figura 4 mostra o cross-plot entre a permeabilidade e a porosidade dos plugs de acordo com o modelo das litofácies. Observa-se que esse modelo não distingue o comportamento petrofísico, de modo que uma mesma litofácie apresenta uma natureza permo-porosa dispersa. A este resultado atribui-se o fato de que a divisão em litofácies carbonáticas é baseada nas propriedades deposicionais, independente do efeito da diagênese. Assim a determinação das zonas petrofísicas foi baseada, inicialmente, nas descrições das litofácies, no entanto, os efeitos da diagênese das rochas também foram levadas em consideração. Em algumas regiões as fácies grainstones foram identificadas parcial ou totalmente cimentadas por carbonato de cálcio e podendo ainda ser encontrada dolomitizada em diferentes intensidades. Observa-se, assim, que a zona grainstone possui as melhores propriedades petrofísicas, seguida pelas zonas packstone e a cimentada, respectivamente. Neste contexto é possível identificar que na região de reservatório, a zona grainstone possivelmente possui uma melhor capacidade de produção (Figura 5). 
$\mathrm{Na}$ tentativa de entender melhor o meio permo-poroso da zona grainstone, o mesmo foi então dividido buscando separar as regiões de acordo com o seu potencial petrofísico. Foram utilizados quatro conceitos distintos, tais como a divisão por litofácies, indicadores IQR (Reservoir Quality Index) e IZF (Flow Zone Index), zonas petrofísicas (ZP), unidades de fluxo (UF) e zonas de fluxo (ZF) (Amaefule et al., 1993).

A Figura 6 ilustra a relação entre a porosidade e a permeabilidade dos plugs discretizadas desta vez pelas UF, que, por meio de uma regressão linear, foram representadas por equações de reta. Comparando essa figura com o modelo proposto por Winland da Figura 7, foi possível estimar os rock types e assim correlacionar as UF com as características petrofísicas correspondentes. A UF1 corresponde aos piores rock types que podem ser correlacionados com os menores raios de gargantas de poro. Em seguida as UF 2 e 3 correspondem aos rock types de qualidade petrofísica intermediaria e, por fim, as UF 4, 5, 6, 7 e 8 mostraramse como as de melhor rock type.

O gráfico estratigráfico de Lorenz modificado foi obtido através dos valores de porosidade e permeabilidade de laboratório. De acordo com essa metodologia, cada ponto de inflexão separa uma potencial zona de fluxo. A Figura 8 mostra o gráfico de Lorenz modificado (esquerda) e na ordem decrescente de acordo com o potencial de fluxo (direita). Os maiores ângulos de inclinação das retas representam as zonas como maior potencial de fluxo. As ZF puderam ser classificadas de acordo com os rock types adaptando o modelo proposto por Winland (1972). Para isto foi necessário trabalhar com as médias aritméticas das porosidades e das permeabilidades dos plugs de cada zona, mostrando a relação entre as ZF, as litofácies e os correspondentes rock types. Assim, as ZF 1 e 9 correspondem a zonas de pior rock type, as ZF 2, 3, 5 e 7 correspondem as zonas intermediárias em quanto que as ZF 4, 5 e 6 correspondem aos melhores rock types (Figura 9).

Através de dados petrofísicos foi possível identificar um modelo que prevê as zonas de fluxo do poço. No entanto, para comparar a efetividade deste método, a curva do perfil de produção PLT foi confrontada a curva de ZF, como mostra a Figura 10. Observou-se que a curva do perfil PLT e a curva KH (permeabilidade $x$ profundidade, a capacidade de fluxo) do método ZF são similares e podem ser correlacionados. Determinadas as distintas zonas de fluxo do reservatório, os dados de perfil puderam ser combinados com os dados de laboratório, de modo a estimar as propriedades petrofísicas de cada zona ao longo da região de reservatório do poço P2.

A Figura 11 mostra os perfis convencionais e o perfil NMR na região de reservatório. Para determinar os volumes de FFI e BFT, a curva de distribuição $T_{2}$ deve ser analisada de modo a encontrar os melhores valores de $T_{2 \text { cutoff. }}$ Assim, a primeira metodologia utilizada contou com os valores de $T_{2 \text { cutoff }}$ obtidos pela NMR de laboratório, esses valores foram estendidos para representar cada ZF, como mostra a curva $T_{2 \text { cutofflab }}$ do track 6 (Figura 12). A segunda metodologia utilizada para separar os volumes de FFI e BFT foi pela média geométrica da distribuição de $\mathrm{T}_{2}$. Esta curva chama-se
$\mathrm{T}_{2 \mathrm{gm}}$ e pode ser vista no track 6 . Os tracks 4 e 5 mostram a relação entre os diferentes cortes de $T_{2}$ e as respectivas curvas de $S_{\text {wir }}$ geradas com base no conceito da NMR. Estas curvas foram comparadas com os valores de $S_{\text {wir }}$ obtidos em laboratório e não mostraram bons ajustes. Assim, no intuito de obter os melhores valores de

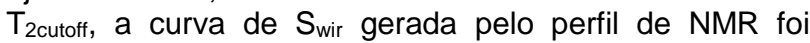
ajustada com os valores de $S_{\text {wir }}$ de laboratório e os correspondentes cortes gerados foram registrados pela curva $T_{2 \text { ccutoffpoço }}$ mostrada no track 6 .

A porosidade total do NMR para todo o poço foi comparada com os dados dos plugs de testemunho, como mostra o track 3 da Figura 13. Foi possível obter uma ótima correspondência entre dados de porosidade de laboratório e dados do NMR de poço. A porosidade referente aos fluidos presos por capilaridade foi obtida para cada zona de fluxo do poço de acordo com seus cortes de $\mathrm{T}_{2}$ como mostra o track 5 . O track 4 mostra os valores de macro, meso e micro porosidade obtidos em laboratório. Verifica-se que os valores correspondentes a meso porosidade são baixos e variam por volta de 0.01 dec ao longo da região de reservatório e que os valores de macro e meso porosidade alternam de modo que seus valores variam em torno de $0.1 \mathrm{dec}$, A micro, meso e macro porosidade NMR foram calibradas através dos dados de laboratório como mostram os tracks 6, 7, 8 e 9 . Os bins correspondentes variaram entre 0,3 a $87 \mathrm{~ms}$ para a micro porosidade, 87 a $110 \mathrm{~ms}$ para meso e 110 a 3000 ms para a macro porosidade. No entanto observou-se que em determinados pontos destas curvas não houve bons ajustes com os dados de testemunho, isso ocorreu devido a variação dos bins não ser feita por ZF, mas sim para todo o reservatório.

A Figura 14 mostra três diferentes metodologias usadas para inferir a permeabilidade ao longo do poço. A permeabilidade Timur/Coates mostrada no track 4, com o coeficiente $\mathrm{C}$ e as exponenciais calibradas por regressão linear, através dos dados de permeabilidade de testemunho, a qual foi realizada para cada zona do poço. A permeabilidade rock type, mostrada no track 3 , foi obtida através das equações de reta das unidades de fluxo mostradas no gráfico semilogarítmico. A curva de permeabilidade obtida, baseada no modelo SDR, não apresentou bons ajustes como mostrado no track 5. A este resultado, são atribuídos os valores do $T_{21 m}$ que não corresponderam aos valores de corte adequados e aos coeficientes teóricos utilizados, os quais não foram calibrados para a formação.

\section{Conclusões}

Neste trabalho, um conjunto de dados da Bacia de Campos permitiu identificar os reservatórios em profundidades diferentes dos poços através dos perfis geofísicos de poço convencionais, o que é comum em regiões com movimentação dos evaporitos. Por outro lado, a descrição petrográfica em litofácies, por não considerar o efeito da diagênese, não representou bem as variações permo-porosas do reservatório, porém, a partir dela foi possível considerar a ZP grainstone como a melhor em termos petrofísicos. A metodologia das ZF, então, permitiu dividir o reservatório dos poços P2 e P3 em zonas menores de acordo com os dados de 
porosidade e permeabilidade dos plugs. A distribuição de $\mathrm{T}_{2}$ do NMR de laboratório, porém, permitiu avaliar as condições de fluido preso por capilaridade e, assim, separar os valores de BFT e FFI, assim como, seus consequentes volumes porosos. Analisando essas curvas, foi possível identificar que a $S_{\text {wir }}$ não corresponde ao volume de poro do BFT o que leva a concluir presença de óleo nos poros pequenos, ou seja, $S_{\text {oir, o que leva a }}$ concluir que a rocha possui molhabilidade mista. A melhor forma para obter os valores de $T_{\text {2cutoff }}$ foi pela calibração da curva $S_{\text {wir }}$ do NMR com os de laboratório. A curva de porosidade total não é influenciada pela presença de diferentes fluídos, pois a área abaixo da curva de distribuição $T_{2}$ não é alterada. Contudo, os valores de macro e micro porosidade foram determinados de acordo com os valores de $\mathrm{T}_{2 \text { cutoff. }} \mathrm{A}$ curva de permeabilidade dos poços P2 e P3 foi derivada do modelo de Timur/Coates utilizando os valores de $\mathrm{T}_{2 \text { cutoff }}$ mais adequados, sendo seus coeficientes obtidos por regressão linear. Os valores de $S_{\text {wir }}$ foram facilmente obtidos através do NMR tendo os valores adequados de $\mathrm{T}_{\text {2cutoff, entretanto, a calibração da curva de distribuição }}$ de garganta de poros do MICP com a curva de distribuição de poros do NMR permitiu obter a saturação de fluido irredutível que se mostrou bem ajustada com os dados dos equipamentos de laboratório (centrifuga e membrana). Através da calibração entre MICP e NMR foi gerada uma pseudo-curva de pressão capilar, resultando que as melhores ZF do poço P2 foram as ZF4, ZF6 e ZF8, e do poço P3, ZF2 e ZF4. O poço P1 não foi dividido em ZF por não possuir dados suficientes de plugs. Assim, baseado na continuidade geológica lateral, os dados das ZP do poço P2 foram extrapolados para as respectivas ZP do poço P1 obtendo, desta forma, os valores aproximados de petrofísica por NMR.

\section{Agradecimentos}

À PETROBRAS por ceder os dados de reservatório da Bacia de Campos, à UENF pela infraestrutura computacional e à SENERGY pela licença acadêmica do software Interactive Petrophysics (IP).

\section{Referências}

- Amaefule, J.; Ohen, H.; David, K. \& Peter, L. 1993. Enhanced reservoir description: Using core and log data to identify hydraulic (flow) units and prediet permeability in uneored intervals/wells. Annual SPE conference and exhibition, Houston - Texas, v. 68th, n. SPE26436.

- Bruhn, C.; Gomes, J.; Lucchese Jr., C. and Johann, P. 2003. Campos Basin: reservoir characterization and management - Historical overview and future challenges. Offshore Technology Conference, Houston, Texas, paper OTC 15220.

- Gunter, G.; Finneran, J.; Hartmann, D. \& Miller, J. 1997. Early determination of reservoir flow units using an integrated petrophysical method. Society of Petroleum Engineers, Inc, n. SPE38679.

- Lucia, F. 1999. Carbonate reservoir characterization. Berlin, Springer-Verlag, 226 pp.

- Machado, V.; Frederico, P.; Netto, P.; Bagueira, R.; Boyd, A.; Sousa, A.; Zielinski, L. \& Junk, E. 2011. Carbonate petrophysics in wells drilled with oil-base mud. SPWLA Meeting.
- Tiab, D. \& Donaldson, E. 1996. Theory and practice of measuring reservoir rock and fluid transport properties. Gulf Publishing Company, 97 pp.

- Winland, H. 1972. Oil accumulation in response to pore size changes, weyburn field, saskatchewan. Amoco Production Research Report, n. F72-G25.

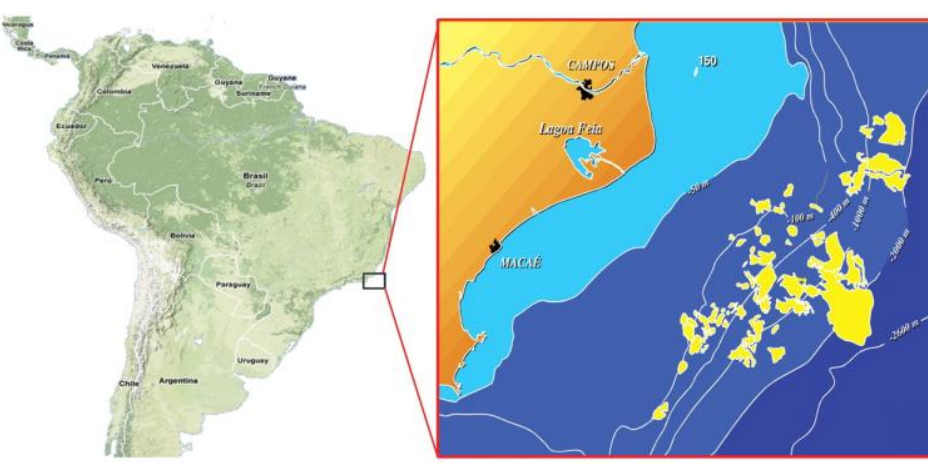

Figura 1. Localização da Bacia de Campos.

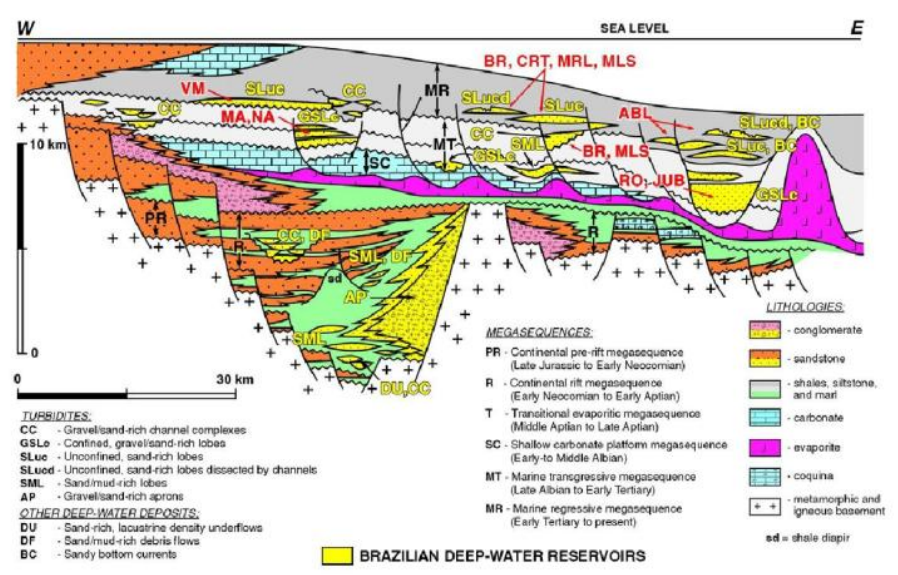

Figura 2. Seção geológica da Bacia de Campos (modificado de Bruhn et al., 2003). 

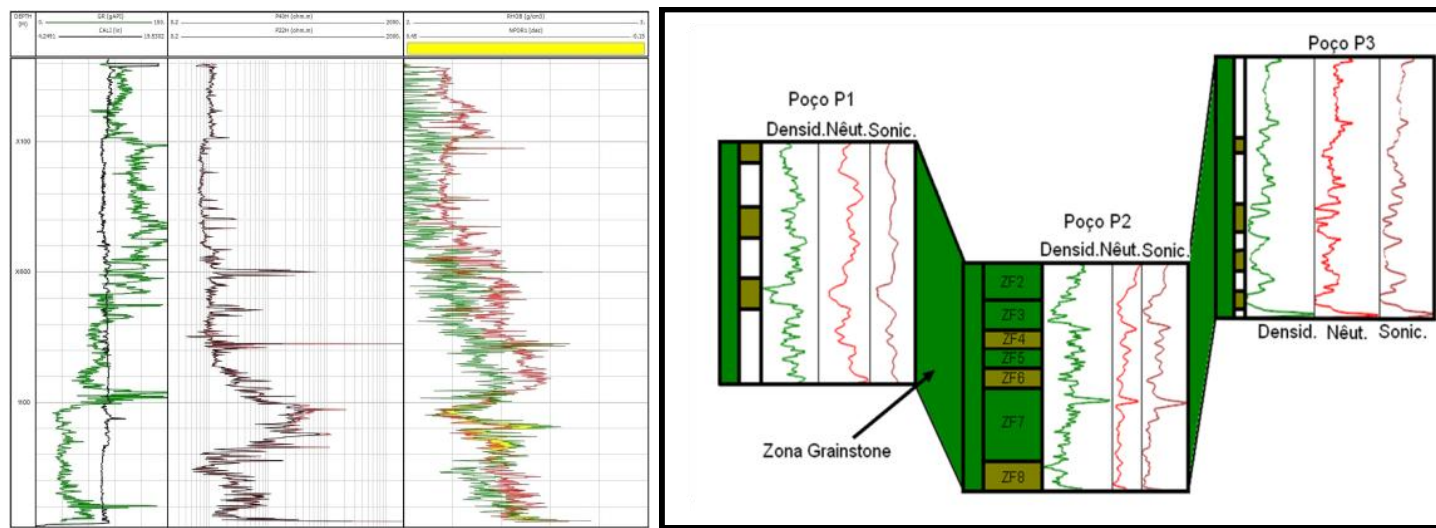

Figura 3. Perfis convencionais ao longo do poço E2.

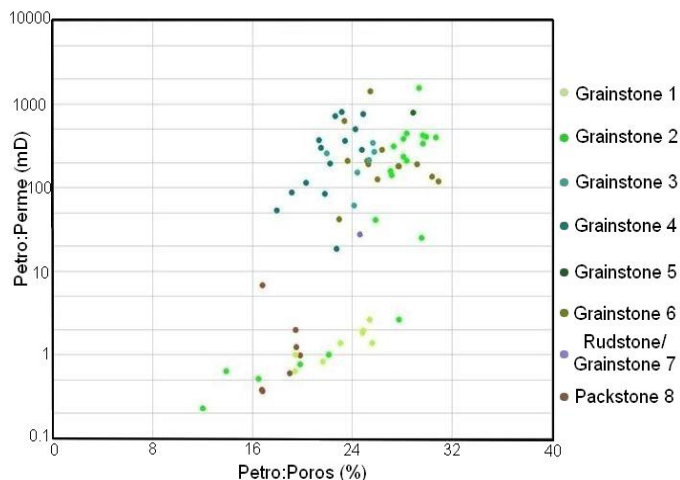

Figura 4. Cross-plot permeabilidade - porosidade dos plugs de acordo com as litofácies.

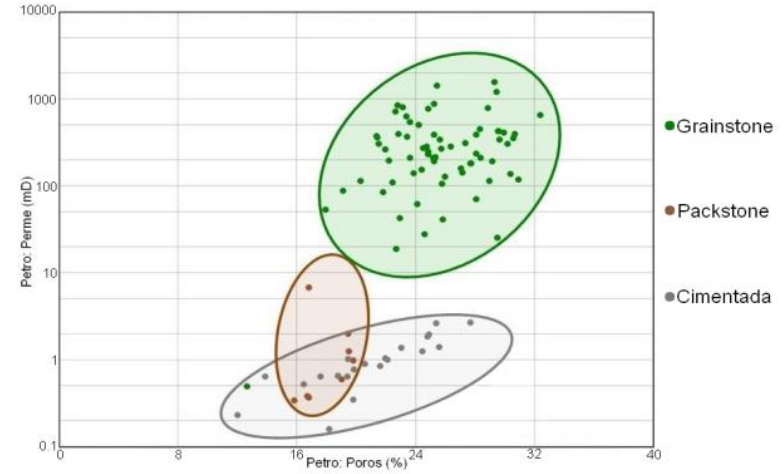

Figura 5. Relação entre a porosidade e a permeabilidade dos plugs.

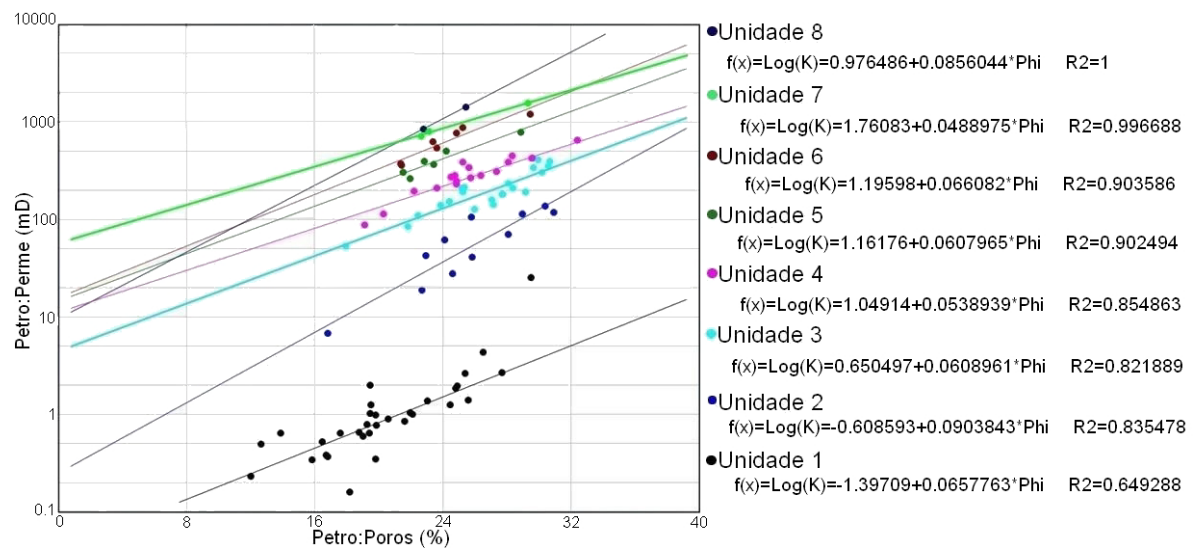

Figura 6. Relação entre a porosidade e a permeabilidade dos plugs discretizados pelas UF, que por meio de regressão linear, foram representadas pelas equações de reta.

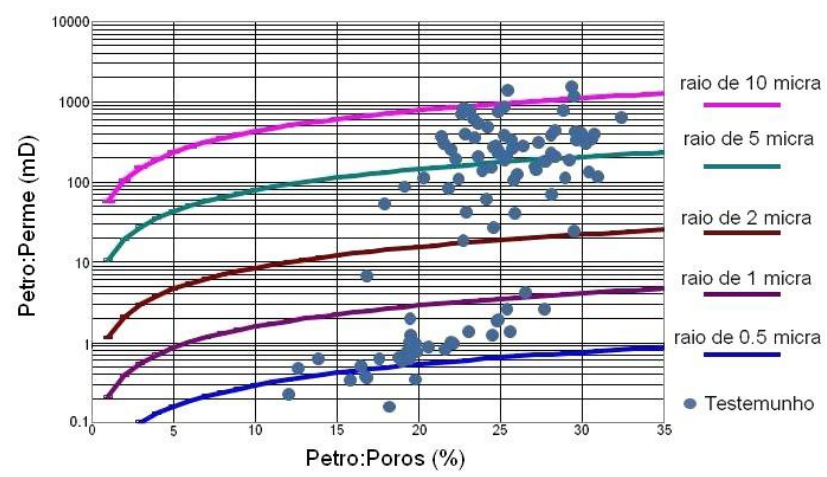

Figura 7. Relação entre K- e o modelo de Winland.

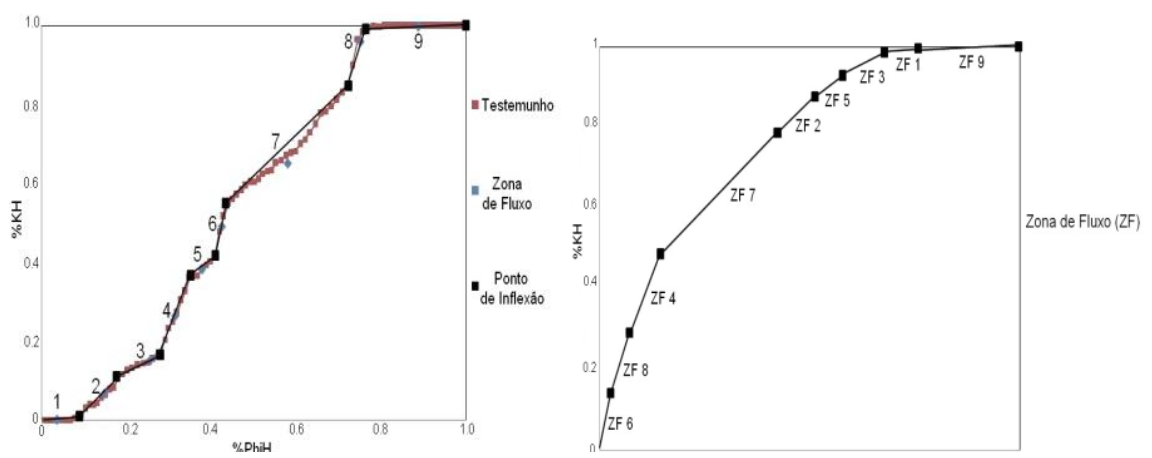

Figura 8. Gráfico estratigráfico de Lorenz modificado e Potencial de fluxo das ZF. 


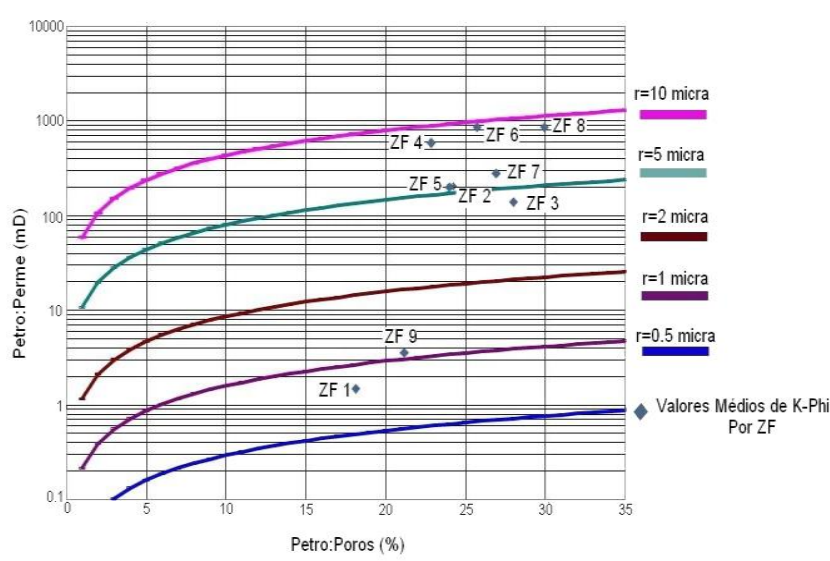

Figura 9. Gráfico de Winland e os rock types.

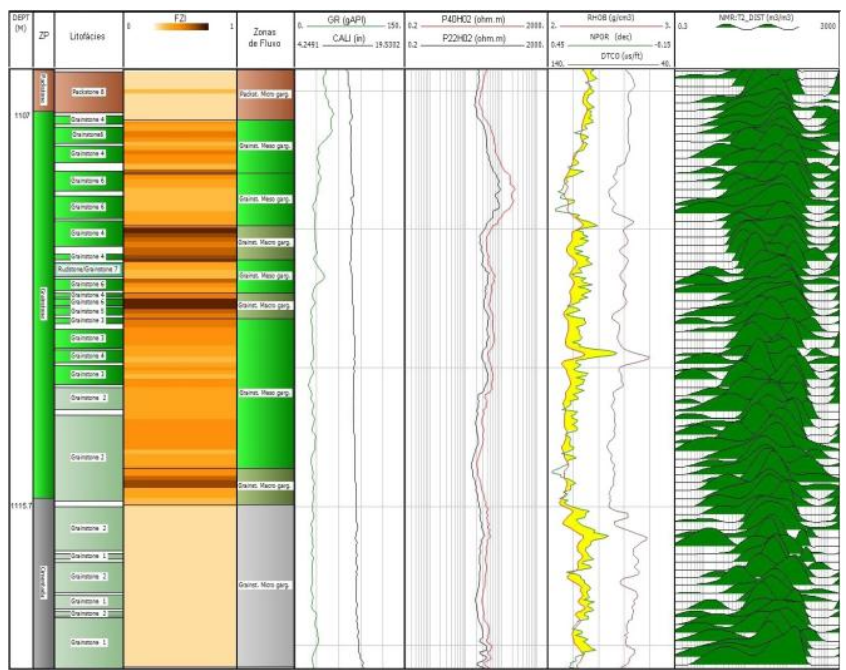

Figura 11. Perfis convencionais e de RMN na região reservatório do poço $\mathrm{P} 2$.

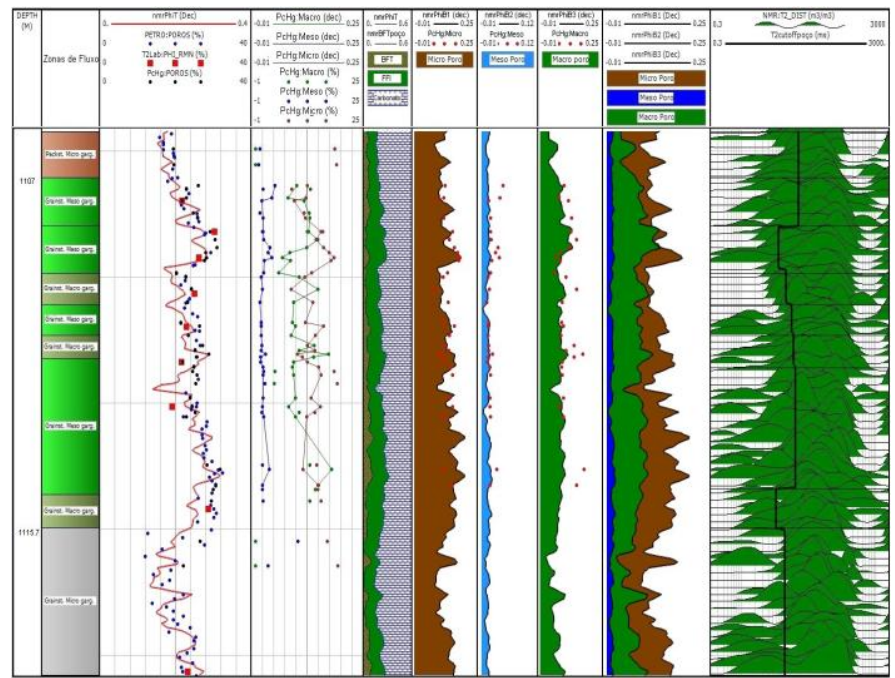

Figura 13. Perfis de porosidade do poço P2.

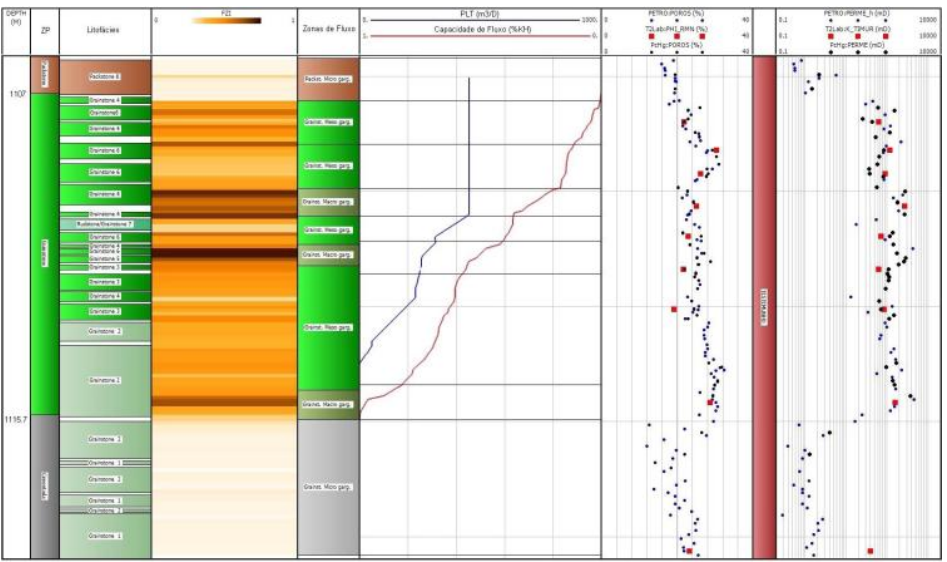

Figura 10. Perfil correlacionando curvas PLT-\%KH do poço P2.

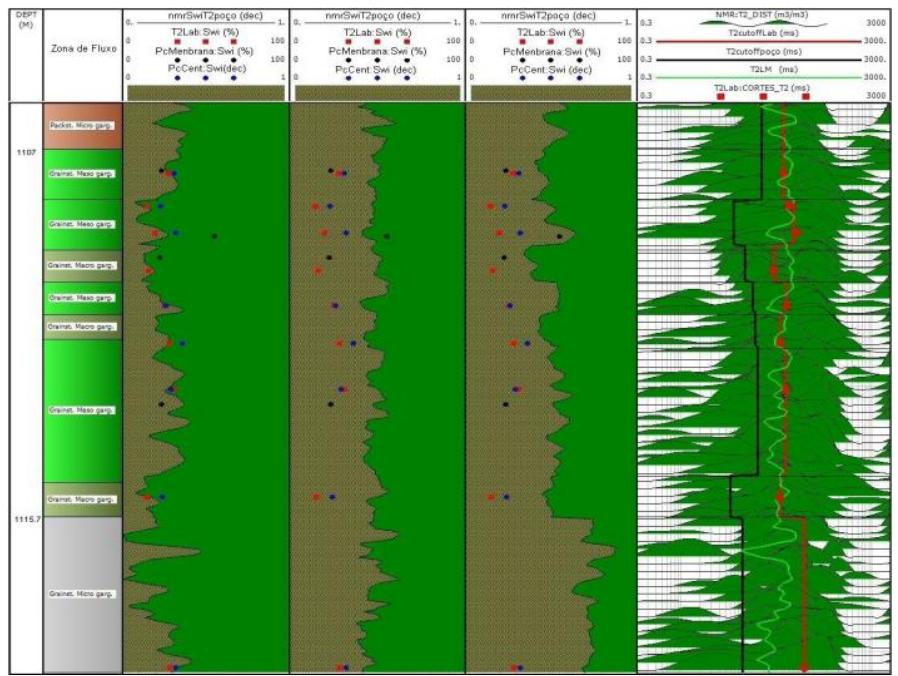

Figura 12. Perfil mostrando diferentes metodologias de obtenção do $S_{\text {wirr }}$.

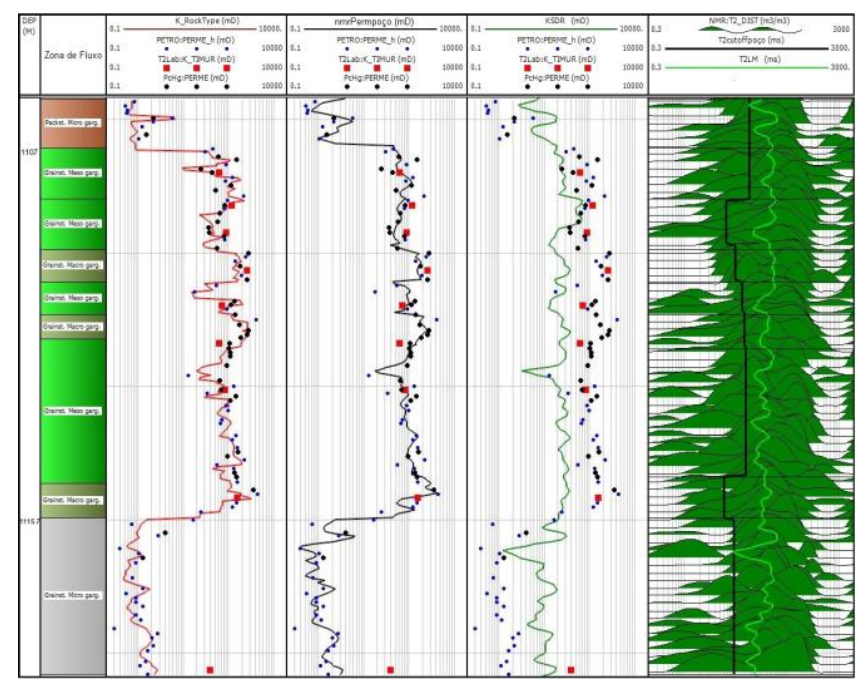

Figura 14. Perfis de permeabilidade do poço E2 obtidos por diferentes metodologias. 